\title{
Nonlinear Phenomena in
}

\section{Stellar Variability}

Edited by M. Takeuti and J.-R. Buchler

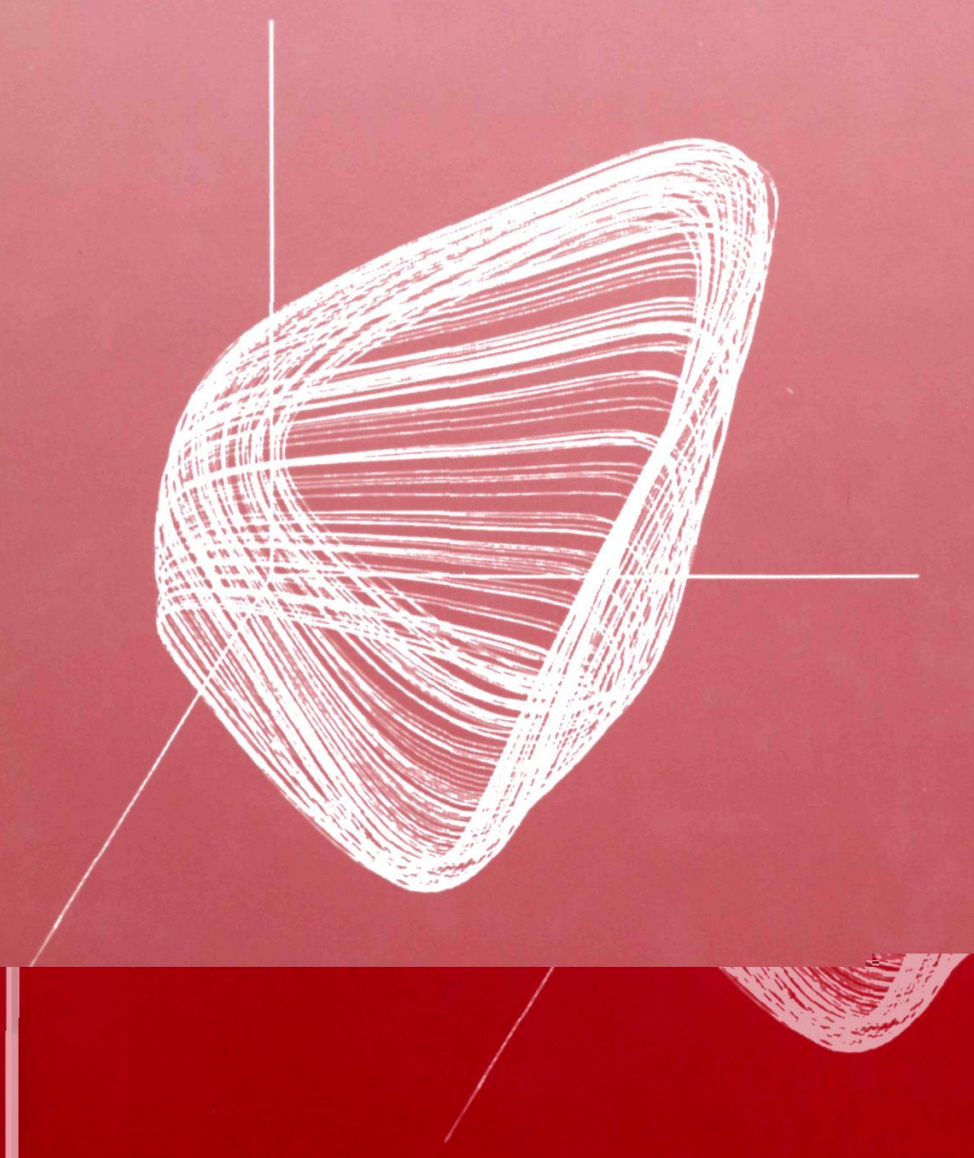




\title{
NONLINEAR PHENOMENA IN STELLAR VARIABILITY
}

\author{
Edited by \\ M. TAKEUTI \\ Astronomical Institute, Tôhoku University, \\ Sendai, Japan \\ AND \\ J.-R. BUCHLER \\ University of Florida, \\ Gainsville, U.S.A.
}

Reprinted from Astrophysics and Space Science

Volume 210, Nos. 1-2, 1993

KLUWER ACADEMIC PUBLISHERS

DORDRECHT / BOSTON / LONDON 
A C.I.P. Catalogue record for this book is available from the Library of Congress.

ISBN 0-7923-2769-1

Published by Kluwer Academic Publishers, P.O. Box 17, 3300 AA Dordrecht, The Netherlands.

Kluwer Academic Publishers incorporates the publishing programmes of

D. Reidel, Martinus Nijhoff, Dr W. Junk and MTP Press.

Sold and distributed in the U.S.A. and Canada by Kluwer Academic Publishers,

101 Philip Drive, Norwell, MA 02061, U.S.A.

In all other countries, sold and distributed by Kluwer Academic Publishers Group, P.O. Box 322, 3300 AH Dordrecht, The Netherlands.

Printed on acid-free paper 


\section{TABLE OF CONTENTS}

IAU Colloquium No. 134 - Nonlinear Phenomena in Stellar Variability

vii

Preface

ix

M. TAKEUTI / Introduction

\section{FUNDAMENTAL THEORIES}

J.R. BUCHLER / A Dynamical Systems Approach to Nonlinear Stellar Pulsations

E.A. SPIEGEL / Patterns of Aperiodic Pulsation

M. AUVERGNE, A. BAGLIN and M.J. GOUPIL / Characterisation of the Dynamics of a Variable Star

H. SAIO / An Overview of Stellar Pulsation Theory

A.S. BARANOV / Nonlinear Oscillations and Beats in the Beta Canis Majoris Stars

W. UNNO and D.-R. XIONG / One Zone Modeling of Irregular Variability of Stellar Convective Envelope

S. MINESHIGE / Accretion Disk Instabilities

Y. NAKAMURA and T. ISHIZUKA / Motion of a Charged Particle around a Black Hole Permeated by Magnetic Field and Its Chaotic Characters

M. YOKOSAWA / Nonlinear Expansion Triggered by Magnetic Stress in Accretion Flow onto a Black Hole

R. KANETAKE, T. TAKESHIMA, K. MAKISHIMA and M. TAKEUTI / Variability of $X$-Ray Emission from Cen X-3 Observed with Ginga

K. TAINAKA, S. FUKAZAWA, H. NISHIMORI, M. YOKOSAWA and S. MINESHIGE / Spatial Pattern Formation of Interstellar Medium

\section{OBSERVATIONAL FACTS}

J.R. PERCY / Supergiant Variables: Recent Observational Results

J.R. PERCY and J.A. MATTEI / The AAVSO Database of Variable Star Observations

D. BARTHÉS, Y. TUCHMAN, M.O. MENNESSIER and J.A. MATTEI / Theoretical Modes Fitting on Miras Light Curves

Z. KOLLÁTH / On the Observed Complexity of Chaotic Stellar Pulsation

T. SERRE / Predicting Variable Star Light Curves

T. YANAGITA, H. SATOH and K. SAIJO / Chaotic Behavior and Statistical Analysis of Some Mira and SR Stars

J.O. PETERSEN / Understanding Changes in Period Ratios

J.O. PETERSEN / On the Application of Fourier Decomposition Parameters

S. TAMURA, M. TAKEUTI and J. ZALEWSKI / Line-Profiles of F Supergiant Stars as Candidates of Proto-Planetary Nebulae 
B.-A. YAO, C.-S. ZHANG, D. QIN and J.-H. TONG / Low Amplitude New Type Variable Stars in Globular Clusters

Z.-Y. YU / Stellar Pulsation: (I) Analysis of Stability of Envelope

Z.-Y. YU/ Stellar Pulsation: (II) Multiple Distinct Shells

M. BREGER / Long-Term Amplitude and Period Variations of Delta Scuti Stars: A Sign of Chaos?

E. MICHEL, M.J. GOUPIL, Y. LEBRETON and A. BAGLIN / The Delta Scuti Star GX Pegasi: A Theoretical Investigation of Its Power Spectrum

M. PAPARÓ, J. PENA, R. PENICHE, C. IBANOGLU, Z. TUNCA and S. EVREN / ShortTerm Amplitude Variation of FM Com (=HR 4684)

S.-Y. JIANG / Period Variations and Evolution of Delta Scuti Variables

Z.-P. LI / Physical Characters of HD 93044

Z.-L. LIU / Period Analysis of the Delta Scuti Star HD 93044

B. PFEIFFER, G. VAUCLAIR, N. DOLEZ, M. CHEVRETON, J.R. FREMY, G. HERPE, M. BARSTOW, S.J. KLEINMAN, T.K. WATSON, J.A. BELMONTE, S.O. KEPLER, A. KANAAN, O. GIOVANNINI, R.E. NATHER, D.E. WINGET, J. PROVENCAL, J.C. CLEMENS, P. BRADLEY, J. DIXSON, A.D. GRAUER, G. FONTAINE, P. BERGERON, F. WESEMAEL, C.F. CLAVER, T. MATZEH, E. LEIBOWITZ and P. MOSKALIK / Observation of a Variable, ZZ Ceti White Dwarf: GD 154

H. SATOH, K. SAIJO and T. YANAGITA / Observation and Statistical Analysis of ZZ Piscium

D.W. KURTZ / Nonlinear, Nonradial Pulsation in Rapidly Oscillating Ap Stars

S.-Y. JIANG / Period Variation of BW Vul

E. KAMBE, H. ANDO and R. HIRATA / Nonradial Oscillations in Zeta Ophiuchi in 1991

T. KOGURE, M. MON and M. SUZUKI / The Onset of Quasi-Periodic Variations in Be Stars

A. OKAZAKI / Optical Observations of FY Persei

M.J. GOUPIL / Some Insights into Stellar Structure from Nonlinear Pulsations

E. ANTONELLO, L. MARASCHI and O. CITTERIO / Capabilities of the Optical Monitor for the Research in X-Ray Source and Stellar Variability

G. L. ISRAELIAN / Wind Variability of LBV Stars

M. MATSUOKA / Variability in Active Galactic Nuclei

\section{MODELS}

Y.A. FADEYEV / Fourier Analysis of the Hydrodynamic Limit-Cycle Models of Pulsating Stars

T. AIKAWA / Bifurcation in Hydrodynamic Models of Stellar Pulsation

G. KOVÁCS / Double-Mode Stellar Pulsation

P. MOSKALIK and J.R. BUCHLER / Period Doubling with Hysteresis in BL Her-Type Models

J.A. GUZIK and A.N. COX / Nonlinear RR Lyrae Models with New Livermore Opacities 
C.G. DAVIS / The Importance of Radiative Transfer in Stellar Pulsation Models

D.D. SASSELOV / Hydrodynamics and Multi-Level Non-LTE Radiative Transfer in Pulsating Atmospheres: Cepheids

E. ANTONELLO / New Opacities and First Overtone Mode Cepheids 331

Y. TANAKA / Coupled Oscillators 333

T. NAKAHARA and Y. TANAKA / An Oscillator Model for Stellar Variability 343

F. YAMAKAWA, T. ISHIDA and M. TAKEUTI / The Coupling Coefficients of Pulsation for Radiative Stellar Models

T. ISHIDA, R. TAKANO, F. YAMAKAWA and M. TAKEUTI / The Coupling Coefficients of Radial Pulsation in Third Order

J. ZALEWSKI / The Role of Convection in Reducing Nonadiabaticity and Mode Coupling in Cepheids

M. SAITOU / The Effect of Convection in Nonlinear One-Zone Stellar Models

Y. OSAKI, M. HIROSE and S. ICHIKAWA / A Unified Model of Dwarf Nova Outbursts Based on the Disk Instability

T. OKUDA and S. MINESHIGE / Pulsational Instability of Accretion Disks around Compact Objects

F. HONMA, R. MATSUMOTO and S. KATO / Numerical Simulations of Pulsationally Unstable Accretion Disks around Supermassive Black Holes

A.T. OKAZAKI / Long-Term V/R Variations of Be Stars Due to Global One-Armed Oscillations of Equatorial Disks

Z.-Y. ZHANG and J.-S. CHEN / A Simplified Model for a Nonlinear Tidal Effect on Accretion Disks in CVs

List of Participants 